\title{
Rotulagem, Segurança Alimentar e Nutricional e Políticas Públicas: uma análise bibliométrica
}

Labeling, Food and Nutritional Security and Public Policies: a bibliometric analysis

Etiquetado, Seguridad Alimentaria y Nutricional y Políticas Públicas: un análisis bibliométrico

Étiquetage, Sécurité Alimentaire et de la Nutrition et Politiques Publiques: une analyse bibliométrique 
${ }^{1}$ Formada em Engenharia de Alimentos, mestranda em Administração Pública e Assistente em Administração na Universidade Federal de Goiás, Goiania, Goiás, GO, Brasil.

E-mail: analuizamc02@gmail.com

${ }^{2}$ Mestranda n Programa de Pós-Graduação em Administração Pública da Universidade Federal de Goiás, Goiânia, GO, Brasil.

E-mail: beatrizcarvalhobarreto@gmail.com

${ }^{3}$ Graduação em Administração pela Universidade Federal do Rio Grande do Sul, Porto Alegre, RS, Brasil, Mestrado em Administração pela Universidade Federal de Uberlândia, Uberlândia, MG, Brasil, e Doutorado em Psicologia pela Pontifícia Universidade Católica de Goiás, Goiânia, GO, Brasil. Atualmente é professor da Universidade Federal de Goiás, Catalão, GO, Brasil, onde coordena o Mestrado Profissional em Administração Pública e dirige o Grupo de Pesquisa M2C - Estudos de Mercado, Consumo e Saúde.

E-mail: solon@ufg.br

4 Graduado em Administração pela Universidade Evangélica e em Engenharia Elétrica pela Universidade Federal de Goiás, Goiânia, GO, Brasil, Mestrado em Administração pela Oklahoma City University, Oklahoma, Estados Unidaos da América, e Doutorado em Engenharia da Produção pela Universidade Metodista de Piracicaba, Piracicaba, SP, Brasil. É professor Adjunto e docente no Programa de PósGraduuação em Administração Pública da Universidade Federal de Goiás e professor da Universidade Estadual de Goiás, Goiânia, GO, Brasil. É líder do Grupo de Pesquisa em Estratégia e Marketing.

E-mail: eliseu@ufg.br 


\section{Resumo}

Abstract
O conceito de segurança alimentar e nutricional surgiu após a Segunda Guerra Mundial, com vistas à uma alimentação em quantidade suficiente, com qualidade e regular. Alguns aspectos como o super processamento dos alimentos, advindos da intensa industrialização vivenciada nos anos pós 1960, influenciou drasticamente a alimentação no mundo. Sem dúvida, atualmente os produtos alimentícios chegam a todas as regiões do mundo, mas questiona-se o quanto esse alimento é saudável (Abramovay, 2017). Essa pesquisa investigou o cenário da produção de artigos, em português e em inglês, nos últimos cinco anos, relacionado às temáticas de: rotulagem, segurança alimentar e nutricional e políticas públicas. Foi realizada uma análise bibliométrica do tipo exploratória, com método quantitativo, desenvolvido por meio de pesquisa do tipo documental. Observou-se que boa parte do número de pesquisas, que abrangem a área de rotulagem, está ligada a alguma outra área como: agrotóxicos, alimentos transgênicos e orgânicos. Isso pode explicar o baixo número de publicações selecionadas para análise, visto que, o objetivo era compreender o cenário da pesquisa das políticas públicas de rotulagem na segurança alimentar e nutricional. É possível inferir que há margem para o crescimento da área de rotulagem voltada para a segurança alimentar e nutricional com foco na promoção de novas políticas públicas que possam combater as doenças crônicas não transmissíveis (DCNT).

Palavras-Chave: Políticas Públicas; Segurança Alimentar e Nutricional. Rótulos; Rotulagem; DCNT.

The concept of food and nutritional security emerged after the Second World War, with a view to a sufficient quantity, with quality and regular food. Some aspects, such as the over-processing of food, resulting from the intense industrialization experienced in the post-1960s, drastically influenced food in the world. Undoubtedly, food products currently reach all regions of the world, but it is questioned how healthy this food is (Abramovay, 2017). This research investigated the scenario of the production of articles, in Portuguese and in English, in the last five years, related to the themes of: labeling, food and nutrition security and public policies. An exploratory bibliometric analysis was performed, using a quantitative method, developed through documentary research. It was observed that a large part of the number of surveys, covering the labeling area, is linked to some other area, such as: pesticides, transgenic and organic foods. This may explain the low number of publications selected for analysis, since the objective was to understand the research scenario of public policies on labeling in food and nutrition security. It is possible to infer that there is scope for growth in the area of labeling focused on food and nutritional security, with a focus on promoting new public policies that can combat chronic non-communicable diseases (NCDs).

Keywords: Public policy; Food and nutrition security; Labels; Labeling; NCDs. 
Resumen

Resumé
El concepto de seguridad alimentaria y nutricional surgió después de la Segunda Guerra Mundial, con miras a una cantidad suficiente, con alimentos de calidad y regulares. Algunos aspectos, como el sobreprocesamiento de los alimentos, resultado de la intensa industrialización vivida en la década posterior a los sesenta, influyeron drásticamente en los alimentos en el mundo. Sin duda, los productos alimenticios llegan actualmente a todas las regiones del mundo, pero se cuestiona lo saludable que es este alimento (Abramovay, 2017). Esta investigación indagó en el escenario de la producción de artículos, en portugués y en inglés, en los últimos cinco años, relacionados con los temas de: etiquetado, seguridad alimentaria y nutricional y políticas públicas. Se realizó un análisis bibliométrico exploratorio, utilizando un método cuantitativo, desarrollado a través de la investigación documental. Se observó que gran parte del número de encuestas, que cubren el área de etiquetado, está vinculada a alguna otra área, como: plaguicidas, alimentos transgénicos y orgánicos. Esto puede explicar el bajo número de publicaciones seleccionadas para el análisis, ya que el objetivo era comprender el escenario de investigación de las políticas públicas sobre etiquetado en seguridad alimentaria y nutricional. Es posible inferir que hay margen de crecimiento en el área de etiquetado enfocado en la seguridad alimentaria y nutricional, con un enfoque en promover nuevas políticas públicas que puedan combatir las enfermedades crónicas no transmisibles (ENT).

Palabras clave: Política pública; Seguridad alimentaria y nutricional; Etiquetas; Etiquetado; ENT.

Le concept de sécurité alimentaire et nutritionnelle est né après la Seconde Guerre mondiale, en vue d'une alimentation suffisante, de qualité et régulière. Certains aspects, tels que la sur-transformation des aliments, résultant de l'industrialisation intense vécue dans les années 60, ont considérablement influencé les aliments dans le monde. Sans aucun doute, les produits alimentaires atteignent actuellement toutes les régions du monde, mais on se demande dans quelle mesure ces aliments sont sains (Abramovay, 2017). Cette recherche a enquêté sur le scénario de la production d'articles, en portugais et en anglais, nos cinq dernières années, en lien avec les thèmes de: l'étiquetage, la sécurité alimentaire et nutritionnelle et les politiques publiques. Faith a réalisé une analyse bibliométrique de type exploratoire, avec méthode quantitative, développée à travers une recherche de type documentaire. Notez qu'une grande partie du nombre d'enquêtes, couvrant le domaine de l'étiquetage, est liée à un autre domaine, tel que: les pesticides, les aliments transgéniques et biologiques. Ceci peut expliquer le faible nombre de publications sélectionnées pour analyse, puisque l'objectif était de comprendre le scénario de recherche des politiques publiques sur l'étiquetage en sécurité alimentaire et nutritionnelle. Il est possible de déduire qu'il y a place à la croissance dans le domaine de l'étiquetage 
visant la sécurité alimentaire et nutritionnelle avec un accent sur la promotion de nouvelles politiques publiques capables de lutter contre les maladies chroniques non transmissibles (MCNT).

Mots clés: Politique publique; Sécurité alimentaire et de la nutrition; Étiquettes; Étiquetage; MCNT. 


\section{Introdução}

A segurança alimentar e nutricional vem ganhando importância na agenda internacional dos últimos anos, intervindo nos acordos e compromissos de desenvolvimento dos governos nacionais (Vasconcellos \& Moura, 2018). Entretanto, os aspectos nutricionais não são os únicos fatores que causam grandes impactos numa alimentação saudável.

O desenvolvimento econômico e tecnológico potencializou a produção de alimentos no mundo, o que tem ocasionado a substituição de dietas abundantes em alimentos de origem vegetal pelo consumo exagerado de produtos processados. Tomando como base a alimentação infantil, $95 \%$ dos casos de obesidade infantil, provêm da alimentação inadequada e da falta de atividades físicas, enquanto somente 5\% dos casos são ocasionados por fatores genéticos (Souza e Cols., 2019).

Essa transformação dos hábitos alimentares, observada na sociedade contemporânea, trouxe o surgimento da Teoria da Transição Nutricional. Essa teoria estabelece que o mundo moderno vive um novo contexto, onde o estado de desnutrição foi substituído pelo sobrepeso e a obesidade, devido a qualidade e a quantidade da dieta, associado às mudanças no estilo de vida. O Brasil tem vivido essa realidade com um declínio no número de desnutrição e na prevalência e aumento do índice de obesidade (Carvalho e Cols., 2019).

Considerando que a Organização Mundial da Saúde (OMS) já classificou o sobrepeso e a obesidade como epidêmicos, é incontestável a importância de uma alimentação saudável, completa e variada na prevenção da obesidade e redução do risco de doenças crônicas não transmissíveis (Boog, 1999; Bevilacqua \& Villena, 2019). De acordo com o Guia Alimentar para a População Brasileira (Brasil, 2014), houve redução na desnutrição em crianças, porém ainda há uma desnutrição crônica em grupos vulneráveis de crianças, mulheres, além de indígenas e quilombolas. Ao mesmo tempo, o país enfrenta o crescimento nos números de pessoas com sobrepeso e obesas, acometendo brasileiros de todas as idades. Além disso, as doenças crônicas são consideradas a principal causa de morte em adultos.

A disponibilização de informações adequadas e compreensíveis sobre os aspectos nutricionais, pode contribuir para a promoção da saúde, além de reduzir o risco de doenças relacionadas à alimentação e à nutrição. Neste aspecto, países latino-americanos já contemplam melhorias no sistema de informação dos alimentos, como é o caso do Chile. Foi criada uma relação entre alimentos e nutrientes e os requisitos que os alimentos devem cumprir. As informações saudáveis são descritas e relacionam gordura total, gordura saturada, ácidos graxos trans, sódio, cálcio, caroteno, vitamina A, vitamina C, ácido fólico, ferro, colesterol e fibra dietética (Coutinho \& Recine, 2007)

Neste contexto, este trabalho teve o objetivo de analisar o cenário da produção de artigos, em português e em inglês, nos últimos cinco anos, relacionados às temáticas de: rotulagem, segurança alimentar e nutricional e políticas públicas, pois entende-se que os rótulos de alimentos são os principais canais de informações, podendo colaborar com a escolha de alimentos saudáveis (Fernandes \& Silva, 2012). 


\section{Referencial Teórico}

O conceito de segurança alimentar está relacionado à quantidade, qualidade e regularidade no acesso aos alimentos e surgiu após a II Guerra Mundial, quando grande parte da Europa foi devastada e ficou sem condições de produzir alimentos (Belik, 2003). Segundo a FAO (Food and Agriculture Organization of the United Nations), segurança alimentar existe se todos tiverem acesso físico e econômico a alimentos de forma suficiente, nutritiva e segura (Assão e Cols., 2007; Mellho-Théry, 2019).

No Brasil, foi criado em 2006, o Sistema Nacional de Segurança Alimentar e Nutricional (SISAN), por meio da Lei Orgânica de Segurança Alimentar e Nutricional (LOSAN) (Brasil, 2006a). Conforme Gonçalves e Cols. (2011), a LOSAN relaciona o conceito de segurança alimentar com os atributos da suficiência, estabilidade, autonomia, sustentabilidade equidade e qualidade. Em nível federal, o SISAN engloba a Conferência Nacional de Segurança Alimentar e Nutricional (CNSAN), o Conselho Nacional de Segurança Alimentar e Nutricional (CONSEA) e a Câmara Interministerial de Segurança Alimentar e Nutricional (CAISAN). Os estados e municípios se orientam seguindo as estruturas em nível federal (Machado e Cols., 2018; Vasconcellos \& Moura, 2018).

A partir da Emenda Constitucional $n^{\circ} 64$, de 2010, a alimentação passou a ser uma garantia expressa na Constituição Federal, art. $6^{\circ}$, como direito social (Brasil, 1988). O Decreto $\mathrm{n}^{\circ} 7.272$, de 25 de agosto de 2010, cria o Sistema Nacional de Segurança Alimentar e Nutricional - SISAN com vistas em assegurar o direito humano à alimentação adequada, institui a Política Nacional de Segurança Alimentar e Nutricional - PNSAN, estabelece os parâmetros para a elaboração do Plano Nacional de Segurança Alimentar e Nutricional, e dá outras providências (Castro, 2019). Essas ações, e muitas outras, foram essenciais para a melhoria na qualidade de vida das pessoas. Desse modo, o Brasil, por estar em transição epidemiológica, ou seja, está no processo de superação da fome, precisa considerar em suas políticas de Segurança Alimentar e Nutricional (SAN) aspectos que tratam tanto do quantitativo quanto do qualitativo da alimentação (Machado e Cols., 2018).

O conceito de alimentação saudável é uma interação entre o biológico e o sociocultural. Esse ponto envolve famílias, profissionais de saúde, gestores e formuladores de políticas públicas e setor produtivo de alimentos como atuadores nos fatores determinantes da alimentação. Dessa forma, são ajustadas medidas regulatórias referentes à rotulagem de alimentos e à publicidade com altos conteúdos de açúcar, gordura e sal; que dirigem a alimentação saudável proposto na Política Nacional de Promoção da Saúde e as ações de avaliação nutricional e promoção da saúde do Programa Saúde na Escola, programa conjunto do Ministério da Saúde e do Ministério da Educação (Brasil, 2006b; Brasil, 2007).

O processo de redemocratização que o Brasil viveu a partir dos anos 1980, fortaleceu as políticas públicas. A cada novo programa de governo, são apresentadas inúmeras políticas públicas que traduzem os propósitos daquele governo e visam a propor intervenções públicas que podem solucionar os problemas do país (Souza, 2003).

Dentro do contexto da Administração Pública, as políticas públicas são instrumentos para atender às necessidades dos cidadãos, por meio de um plano de ações que visam a resolver um problema público coletivo. Essas questões servem para pontuar a dificuldade de implantação de política pública, controle de rotulagem e verificação do desempenho de uma ação do governa centrada em SAN (Monteiro, Coutinho \& Recine, 2005). 
Ao todo, considera-se 5 pontos de avaliação, mas a quantidade reduzida não afasta a dificuldade de controle: 1) método da FAO de cálculo da disponibilidade calórica diária per capita; 2) cálculo da renda mínima para consumo alimentar e não alimentar; 3) cálculo do consumo alimentar - como os recordatórios quantitativos das últimas 24 horas, frequência de consumo alimentar ou a quantificação dos gastos familiares com aquisição de alimentos; 4) antropometria e; 5) escalas psicométricas do acesso familiar aos alimentos (Aliaga, 2020). Estranhamente, a questão de rotulagem não consta nos aspectos avaliativos, mesmo o Brasil fazendo parte de um grupo aproximadamente 100 países, que reconhece, seja por meio de marcos legais, normativos e tratados, o direito à alimentação como um direito humano. Esse compromisso busca promover a dignidade na alimentação bem como sua qualidade.

No Brasil, as políticas públicas de segurança alimentar e nutricional são frequentemente de combate à fome e assistencialistas. Além disso, os interesses pessoais de vários segmentos e a estrutura descentralizada do Estado entre o governo federal e os governos subnacionais, são fatores que prejudicam o avanço de políticas públicas de segurança alimentar e nutricional (Cardoso Júnior, 2011).

A rotulagem de alimentos está inserida no Plano Nacional de Segurança Alimentar e Nutricional (Plansan), que define que a Agência Nacional de Vigilância Sanitária (ANVISA) disponibilize, em seu portal eletrônico, informações que facilitem a interpretação dos rótulos de alimentos pelos consumidores, além da revisão das resoluções de rotulagem vigentes (CAISAN, 2018).

A Resolução RDC 259 define rótulo como toda inscrição, legenda ou imagem, escrita, impressa, estampada, gravada em relevo ou colada sobre a embalagem do alimento (Brasil, 2002). Em consonância a isso, o Código de Defesa do Consumidor, em seu artigo $6^{\circ}$, determina que a informação sobre produtos e serviços deve ser clara e adequada e "com especificação correta de quantidade, características, composição, qualidade e preço, bem como sobre os riscos que apresentem" (Brasil, 1990).

O Decreto-lei $\mathrm{n}^{\circ} 986$ de 1969, determina que "todo o alimento será exposto ao consumo ou entregue à venda depois de registrado no Ministério da Saúde" (Brasil, 1969). Este mesmo decreto, ainda em vigor, estabelece a obrigatoriedade de informações como tipo de alimento, nome ou marca, nome do fabricante, local da fábrica, número de registro no Ministério da Saúde, indicação do emprego de aditivos intencionais, número de identificação da partida, lote, data de fabricação e indicação do peso ou volume, que devem constar de forma legível nas embalagens dos produtos (Brasil, 1969).

A Resolução ANVISA RDC 360/2003 - Regulamento Técnico Sobre Rotulagem Nutricional de Alimentos Embalados, determina a informação nutricional obrigatória de valor energético, carboidratos, proteínas, gorduras totais, gordura saturada, gordura trans, fibra alimentar e sódio, partindo de uma dieta de $2000 \mathrm{Kcal}$ ou $8400 \mathrm{KJ}$ diárias (Brasil, 2003; Pereira e Cols., 2019).

Os rótulos dos alimentos podem ser compreendidos como importante instrumento de comunicação, que podem promover escolhas mais adequadas, uma vez que trazem informações importantes para o consumidor, sendo uma questão relevante para a segurança alimentar e nutricional. Trabalhados de forma consciente, com linguagem menos técnica e legibilidade adequada, os rótulos podem contribuir para a redução de gastos pelo sistema de saúde com a obesidade e outras Doenças Crônicas Não Transmissíveis - DCNT (Silva \& Senger, 2014).

O contexto de abundância de alimentos industrializados no mercado mundial, criou ambiente favorável para o uso do marketing como fator de diferenciação para o sucesso das empresas alimentícias (Dantas \& Silva, 2019; Alcantara e Cols., 2018). A situação se torna ainda mais alarmante quando se percebe, por meio de vários estudos, que esse consumo exagerado de produtos industrializados também é alto em crianças (Almeida., 2018; Patzlaff \& Melo, 2020; Landim e Cols, 2020; Lopes, e Cols, 2020), que são mais suscetíveis ao apelo das embalagens lúdicas com personagens e brindes (Versiani, 2020). 
Neste sentido, é necessário reforçar a importância de políticas públicas, que privilegiem ações direcionadas ao consumidor e que o conscientize sobre as informações presentes nos rótulos dos alimentos e, proporcionando ao consumidor condições de optar por uma alimentação saudável. No Brasil, a ANVISA possui uma tarefa extremamente relevante no aspecto da segurança alimentar e nutricional, que é a responsabilidade de fiscalizar e normatizar a rotulagem dos alimentos e as falhas existentes na legislação brasileira de rotulagem (Minuzi, 2019).

\section{Procedimentos Metodológicos}

A preocupação acerca da mensuração do valor científico das publicações deu origem à bibliometria, que é o estudo dos aspectos quantitativos da produção científica e disseminação da informação (Medeiros e Cols., 2015). Dessa forma, é possível visualizar o cenário da produção sobre as políticas públicas de rotulagem de alimentos na segurança alimentar e nutricional e, por isso, este estudo concentra-se na análise bibliométrica do tipo exploratória, com método quantitativo, desenvolvido por meio de pesquisa do tipo documental.

Foi realizada uma pesquisa bibliométrica utilizando como base de dados o portal de periódicos da CAPES e as plataformas Scielo, Scopus e Google Acadêmico (Google Scholar). A busca foi realizada em outubro de 2019 e observou-se a janela temporal dos últimos cinco anos, ao considerar publicações desde 2015, visto que esse período também se refere às publicações mais atualizadas sobre os temas.

No primeiro momento, as palavras-chave utilizadas foram "' Políticas Públicas' AND 'Segurança Alimentar e Nutricional' AND 'Rótulos' AND 'Rotulagem'” e foram encontrados 201 resultados. Em seguida, foi realizada a primeira seleção que eliminou trabalhos de conclusão de curso, dissertações, teses e livros, tendo como resultado 35 artigos. Como também é de interesse deste estudo as publicações em inglês, foram utilizadas as palavras-chave' Public Policy' AND 'food and nutrition security' AND 'labels' AND 'labeling'”. Nesse momento, foram encontrados 76 resultados e após a primeira seleção, utilizando o mesmo critério da seleção anterior, restaram 22 artigos. Após a primeira seleção restaram, considerando artigos em português e em inglês, 57 estudos.

A inclusão das publicações para análise foi definida a partir da leitura dos títulos e resumos dos artigos encontrados, com o foco em selecionar apenas os que realmente se relacionam à temática da rotulagem de alimentos e segurança alimentar e nutricional. Com essa leitura foram eliminados mais alguns artigos que tratavam de diversas temáticas, como por exemplo: agrotóxicos, alimentos transgênicos e orgânicos, entre outras, restando, ao final, 24 artigos. Para ilustrar a metodologia da pesquisa foi criada a Figura 1, que mostra as etapas de pesquisa, seleção e análise dos artigos. 
Figura 1. Fluxograma de pesquisa.

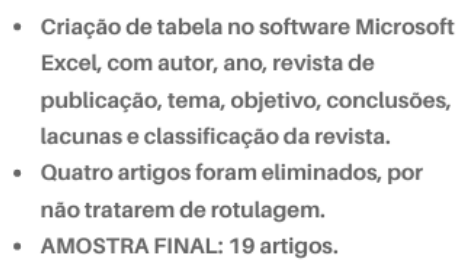

- Criação de tabela no software Microsoft Excel, com autor, ano, revista de publicação, tema, objetivo, conclusões, lacunas e classificação da revista.

- Quatro artigos foram eliminados, por não tratarem de rotulagem.

- AMOSTRA FINAL: 19 artigos.

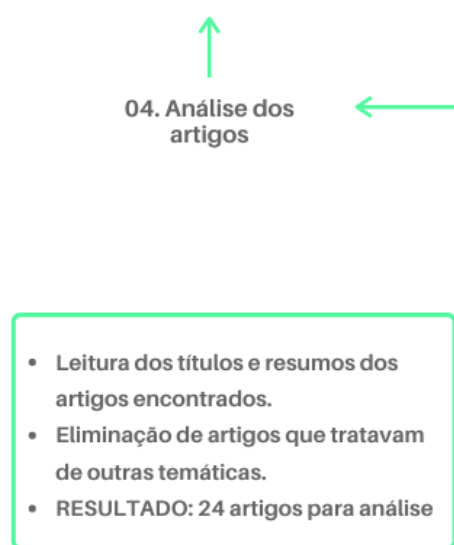

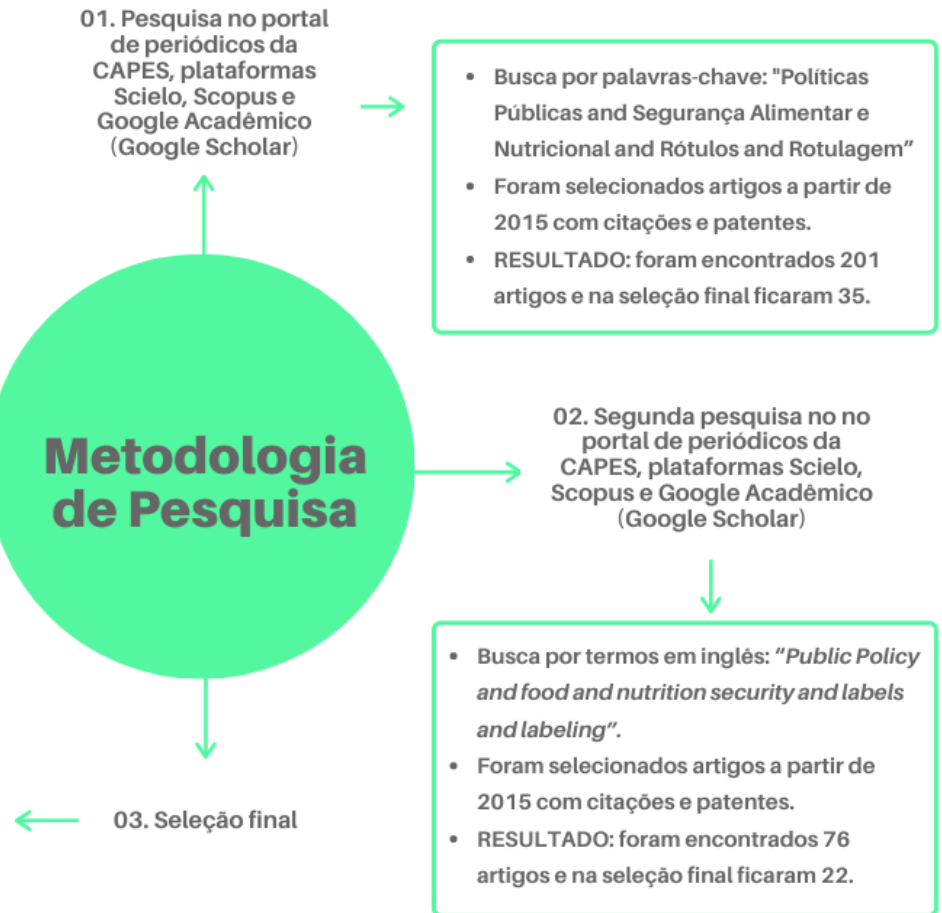

\section{Goiânia, 2020. Fonte: Elaborada pelos autores}

$\mathrm{Na}$ última etapa foi realizada a análise dos artigos por meio da leitura do texto e da interpretação da amostra utilizando uma tabela criada no software Microsoft Excel (Quadro 1). Nesse quadro foram utilizadas as seguintes categorias: autor, ano, idioma, tema, tipo de estudo, revista de publicação e classificação da revista (Qualis), a fim de compreender as tendências de produção e divulgação do conhecimento nas temáticas. Após essa coleta e análise dos dados, outros 5 artigos foram eliminados, por não tratarem de rotulagem. Ao final, restaram 19 artigos. Sendo assim, os critérios de inclusão utilizados foram: recorte temporal (a partir de 2015) e artigos em português e inglês, disponíveis online. E os critérios de exclusão foram: teses, dissertações, livros e artigos que não tratem das temáticas deste trabalho.

\section{Resultados e Discussões}

A amostra do presente estudo correspondeu ao número total de 19 artigos, sendo dezesseis em português e três em inglês. O Quadro 1 foi criado para auxiliar na seleção dos artigos e dar melhor compreensão das tendências de produção das temáticas estudadas. 
Quadro 1. Relação de Publicações

\begin{tabular}{|c|c|c|c|}
\hline AUTORES/ANO/IDIOMA & TEMA DO ARTIGO & REVISTA & QUA \\
\hline Pereira e Cols., 2017 (Pt) & Rotulagem nutricional & $\begin{array}{l}\text { DEMETRA: } \\
\text { Nutrição \& Saúde }\end{array}$ & B3 \\
\hline $\begin{array}{l}\text { Woodhouse \& Woodhouse, } \\
2018 \text { (Ing) }\end{array}$ & $\begin{array}{l}\text { Direito das crianças à } \\
\text { alimentação }\end{array}$ & Family Court Review & $*$ \\
\hline Caivano e Cols., 2017 (Pt) & $\begin{array}{l}\text { Conflitos de interesses } \\
\text { nas estratégias da indústria } \\
\text { alimentícia }\end{array}$ & $\begin{array}{l}\text { DEMETRA: } \\
\text { Nutrição \& Saúde }\end{array}$ & B3 \\
\hline Pereira e Cols., 2019 (Pt) & $\begin{array}{l}\text { Direito às informações } \\
\text { nos rótulos dos alimentos }\end{array}$ & Aletheia & $\mathrm{B} 2$ \\
\hline Hoek e Cols., 2017 (Ing) & $\begin{array}{l}\text { Escolhas alimentares } \\
\text { saudáveis e sustentáveis }\end{array}$ & Food Quality and Preference & A2 \\
\hline Santos. e Cols., 2016 (Pt) & $\begin{array}{l}\text { Educação alimentar e } \\
\text { nutricional (leitura de rótulos) }\end{array}$ & Revista Ciência em Extensão & B3 \\
\hline Marques \& Xavier, $2019(\mathrm{Pt})$ & $\begin{array}{c}\text { Alimentação saudável } \\
\text { com enfoque interdisciplinar }\end{array}$ & $\begin{array}{lll}\text { Brazilian } & \text { Journal of } \\
\text { Development } & & \\
\end{array}$ & B2 \\
\hline $\begin{array}{l}\text { Simon, Ramos \& dos Santos } \\
\text { Rosa, } 2017(\mathrm{Pt})\end{array}$ & $\begin{array}{l}\text { Educação alimentar e } \\
\text { nutricional }\end{array}$ & $\begin{array}{l}\text { DEMETRA: } \\
\text { Nutrição \& Saúde }\end{array}$ & B3 \\
\hline Jesus. e Cols., 2018 (Pt) & $\begin{array}{cc}\text { Importância } & \text { da } \\
\text { Vigilância Sanitária } & \\
\end{array}$ & $\mathrm{GeTeC}$ & $\mathrm{C}$ \\
\hline Davies e Cols., 2017 (Ing) & $\begin{array}{ll}\text { Guia } & \text { Alimentar } \\
\text { Brasileiro } & \\
\end{array}$ & Public Health Nutrition & $\mathrm{A} 2$ \\
\hline Azevedo, $2019(\mathrm{Pt})$ & \begin{tabular}{ll}
\multicolumn{1}{c}{ Interferência } & das \\
indústrias alimentares & nas \\
políticas (lobby alimentar) &
\end{tabular} & Revista Ingesta & Anais \\
\hline Pontes \& Cordeiro, $2018(\mathrm{Pt})$ & $\begin{array}{c}\text { Educação alimentar e } \\
\text { nutricional (leitura de rótulos) }\end{array}$ & $\begin{array}{ccc}\text { Revista } & \text { da } & \text { Associação } \\
\text { Brasileira de Nutrição } & \end{array}$ & $\mathrm{C}$ \\
\hline Backes e Cols., 2019 (Pt) & $\begin{array}{ll}\text { Mudanças } & \text { de } \\
\text { comportamento alimentar } & \\
\end{array}$ & $\begin{array}{ccc}\text { Revista } & \text { Saúde } & \text { e } \\
\text { Desenvolvimento Humano } & \\
\end{array}$ & B4 \\
\hline Ferreira e Cols,.2018 (Pt) & Marketing Nutricional & $\begin{array}{ccc}\text { Revista } & \text { Estratégia } & \mathrm{e} \\
\text { Desenvolvimento } & & \\
\end{array}$ & B4 \\
\hline Valicente, 2016. (Pt) & $\begin{array}{l}\text { Direito às informações } \\
\text { nos rótulos dos alimentos }\end{array}$ & Cadernos de Direito & B3 \\
\hline Santos e Cols., 2017. (Pt) & $\begin{array}{l}\text { Alimentação escolar } \\
\text { adequada para indivíduos } \\
\text { com reações adversas }\end{array}$ & $\begin{array}{l}\text { III Seminário de Tecnologias } \\
\text { Aplicadas em Educação e Saúde }\end{array}$ & Anais \\
\hline Castro, 2016. (Pt) & $\begin{array}{l}\text { Implantação } \\
\text { produtos trangênicos e } \\
\text { desafios }\end{array}$ & \begin{tabular}{lr}
\multicolumn{1}{c}{ Fronteira: Journal of } \\
Social,Technological \\
Environmental Science
\end{tabular} & B3 \\
\hline Magalhães, $2017(\mathrm{Pt})$ & 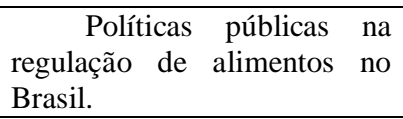 & Revista de Direito Sanitário & B1 \\
\hline Firmino e Cols.,2017. (Pt) & $\begin{array}{ll}\text { Rotulagem } & \mathrm{de} \\
\text { Suplementos } & \\
\end{array}$ & $\begin{array}{l}\text { Oikos: Família e Sociedade em } \\
\text { Debate }\end{array}$ & B3 \\
\hline
\end{tabular}

Goiânia, 2020. Fonte: Elaborado pelos autores

Foram selecionados artigos com ano de publicação a partir de 2015. No ano de 2015, não foram encontradas publicações sobre a temática. Entretanto, em 2016, foram três publicações. O ano de 2017 concentra cerca de $42 \%$ dos artigos encontrados, ou seja, oito artigos, mostrando assim uma tendência de aumento das publicações, que não se manteve, visto que nos anos de 2018 e 2019 observou-se decréscimo, sendo quatro publicações em cada ano, conforme ilustrado no Quadro 2.

Quadro 2. Artigos publicados por ano

\begin{tabular}{|l|l|l|l|l|l|l|}
\hline Ano & $\mathbf{2 0 1 5}$ & $\mathbf{2 0 1 6}$ & $\mathbf{2 0 1 7}$ & $\mathbf{2 0 1 8}$ & $\mathbf{2 0 1 9}$ & Total \\
\hline Artigos & 0 & 3 & 8 & 4 & 4 \\
\hline Porcentagem & $0 \%$ & $16 \%$ & $42 \%$ & $21 \%$ & $21 \%$ & 19 \\
\hline
\end{tabular}

Goiânia, 2020. Fonte: Elaborado pelos autores 
Apesar do declínio nas publicações em 2018 e 2019, a linha de tendência, ilustrada no Gráfico 1, mostra crescimento em comparação aos anos anteriores. Deste modo, vê-se como crescente o interesse dos autores e revistas pelos assuntos de políticas públicas de rotulagem de alimentos e segurança alimentar e nutricional

\section{Gráfico 1. Publicações por ano}

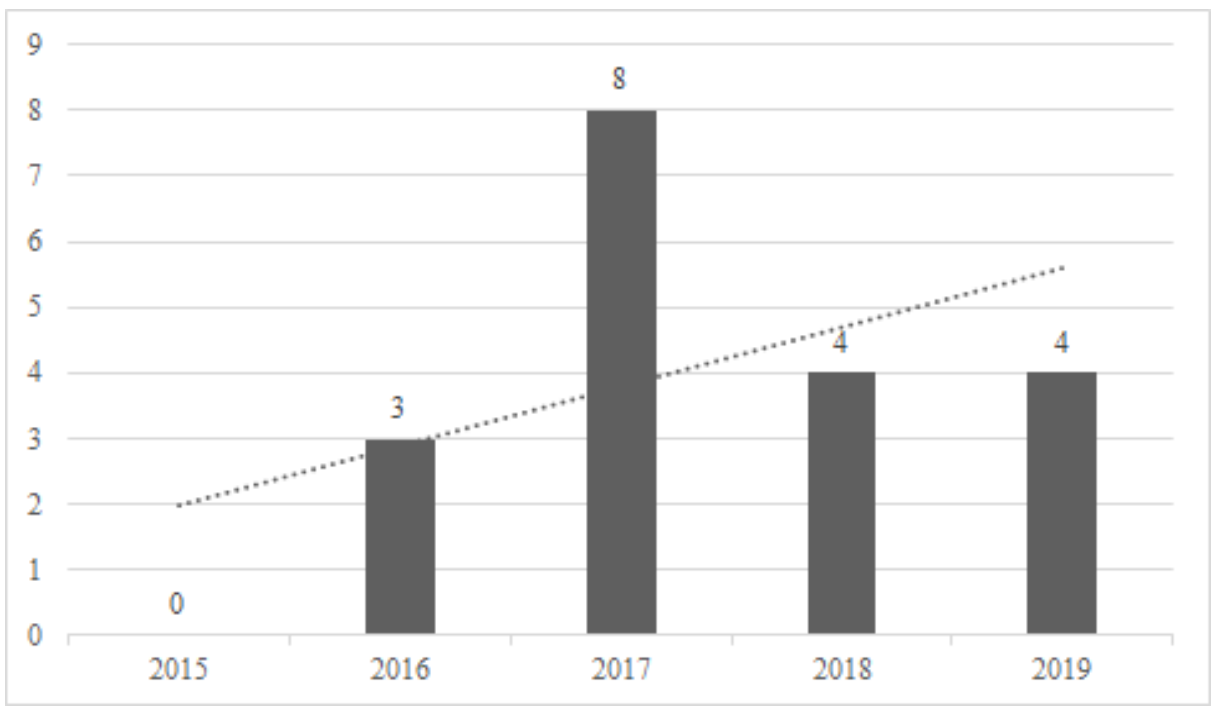

Goiânia, 2020. Fonte: Elaborado pelos autores

Ao analisar as revistas, nas quais foram encontradas as publicações, sem considerar os artigos publicados em anais de evento, a "DEMETRA: Alimentação, Nutrição \& Saúde" se destacou, pois teve o maior número de publicações. Dos 19 artigos, três foram publicados na referida revista, enquanto as outras tiveram somente um artigo. O Quadro 3 mostra a distribuição dos artigos por revista.

\section{Quadro 3. Artigos publicados por revista}

\begin{tabular}{|l|c|}
\hline Nome da Revista & n Publicações \\
\hline DEMETRA: Alimentação, Nutrição \& Saúde & 3 \\
\hline Family Court Review & 1 \\
\hline Aletheia & 1 \\
\hline Food Quality and Preference & 1 \\
\hline Revista Ciência em Extensão & 1 \\
\hline Brazilian Journal of Development & 1 \\
\hline Public Health Nutrition & 1 \\
\hline Revista Ingesta & 1 \\
\hline Revista da Associação Brasileira de Nutrição & 1 \\
\hline Revista Saúde e Desenvolvimento Humano & 1 \\
\hline Revista Estratégia e Desenvolvimento & 1 \\
\hline Cadernos de Direito & 1 \\
\hline III Seminário de Tecnologias Aplicadas em Educação e Saúde & 1 \\
\hline Fronteira: Journal of Social,Technological and Environmental Science & 1 \\
\hline Revista de Direito Sanitário & 1 \\
\hline Oikos: Família e Sociedade em Debate & 1 \\
\hline
\end{tabular}

Goiânia, 2020. Fonte: Elaborado pelos autores 
O Qualis é um sistema brasileiro que classifica a qualidade das pesquisas e dos artigos científicos com base nas revistas e anais onde foram publicados. Essa classificação foi criada pela Capes (Coordenação de Aperfeiçoamento de Pessoal de Nível Superior). Existiam, até o fechamento dessa pesquisa, 8 (oito) extratos de classificação: A1, A2, B1, B2, B3, B4, B5 e C. Qualis A1 e A2 compreendem excelência internacional, B1 e B2, excelência nacional, os Qualis B3, B4 e B5, relevância média e o C, baixa relevância (Erdmann e Cols., 2009). É importante salientar que foi considerada a área de avaliação de Administração Pública ou, para aqueles que não se enquadraram, Interdisciplinar. A revista DEMETRA, que obteve maior número de publicações dentre as encontradas nesta pesquisa, possui classificação de Qualis B3, considerando a área de avaliação de administração pública, o que significa que esses artigos têm relevância média na área.

No Quadro 4, é possível visualizar o número de publicações por Qualis. Considerou-se somente as publicações em revistas, pois dois dos artigos encontrados foram publicados em anais de eventos e uma das revistas não tem classificação no Qualis. Observa-se que a maioria das revistas são classificadas no extrato B do Qualis (B1, B2, B3 e B4), que somadas equivalem a 75\% do total, ou seja, uma pequena parcela das publicações correspondente à excelência nacional e a maioria constituise de relevância média. Tem-se ainda duas publicações dentro de revistas dos Qualis do nível A2, essas são estrangeiras e os artigos escritos em inglês.

\section{Quadro 4. Publicações por Qualis}

\begin{tabular}{|c|c|c|}
\hline Qualis da revista & Número de publicações & Porcentagem \\
\hline $\mathrm{A} 2$ & 2 & $12 \%$ \\
\hline $\mathrm{B} 1$ & 1 & $6 \%$ \\
\hline $\mathrm{B} 2$ & 2 & $12 \%$ \\
\hline $\mathrm{B} 3$ & 7 & $44 \%$ \\
\hline $\mathrm{B} 4$ & 2 & $13 \%$ \\
\hline $\mathrm{C}$ & 2 & $13 \%$ \\
\hline
\end{tabular}

Goiânia, 2020. Fonte: Elaborado pelos autores

Após as análises quantitativas dos artigos encontrados, como o número de publicações por ano, por revista e por Qualis, foi encaminhada a fase de análise de semelhanças. A análise de similitude foi feita por meio do software Iramuteq, realizada a partir do corpus textual dos 19 resumos dos artigos selecionados nesta pesquisa e, para a formação da "árvore", considerou-se somente palavras com mais de sete repetições, a fim de deixar mais clara a visualização das palavras e considerar somente as mais importantes. Deste modo, pode-se visualizar, a partir da Figura 2, a relação entre as palavras e a conectividade nas cinco classes encontradas, sendo essas: rótulo, alimento, nutricional, alimentar e saúde. O agrupamento 'rótulo' evidencia as palavras "conhecimento" e "público", lembrando que os rótulos têm a função de tornar públicas as informações daquele alimento, ao promover mais conhecimento para aqueles que o ingerem. No agrupamento 'alimento', vê-se palavras como "saudável" e "direito", uma vez que consumir alimentos saudáveis é um direito previsto na Constituição Federal. No agrupamento 'alimentar', evidencia-se a presença de "educação" e "escolha". Quando analisado o agrupamento 'nutricional', as principais relações são com "rotulagem”, "consumidor" e "informação", o que confere mais uma vez a ideia da rotulagem nutricional como fonte de informações ao consumidor para o subsídio de escolhas melhores. Por fim, o último agrupamento de "saúde', mostra relação com "promoção" e "ação", o que reforça a ideia de políticas públicas voltadas à segurança alimentar e nutricional. 


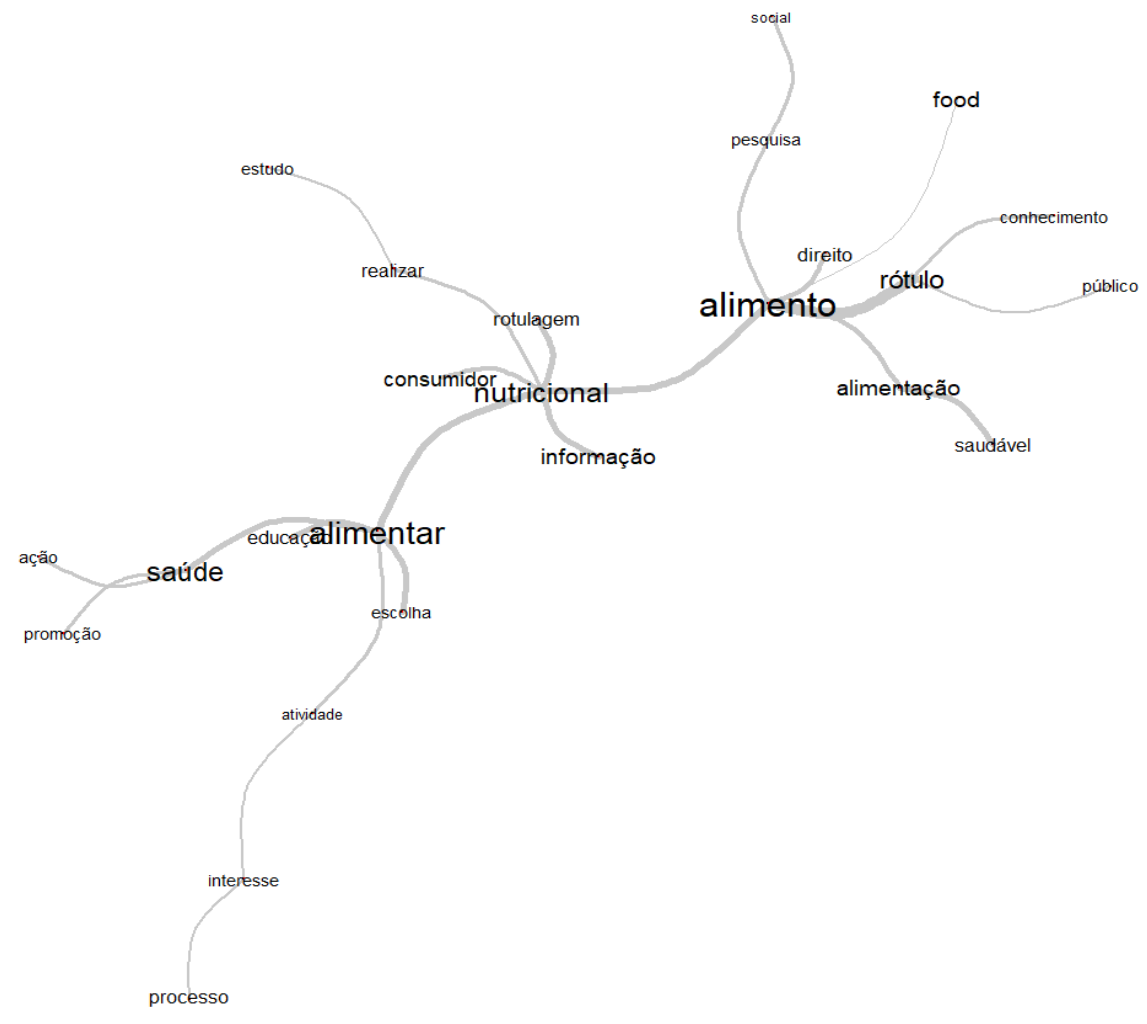

Figura 2. Análise de similitude

Goiânia, 2020. Fonte: Elaborada pelos autores

A análise a seguir apresenta a nuvem de palavras, esboçada na Figura 3. Essa técnica agrupa as palavras e organiza em função da representatividade e importância, ou seja, quanto maior a palavra, mais importante é para aquele grupo de artigos analisados.

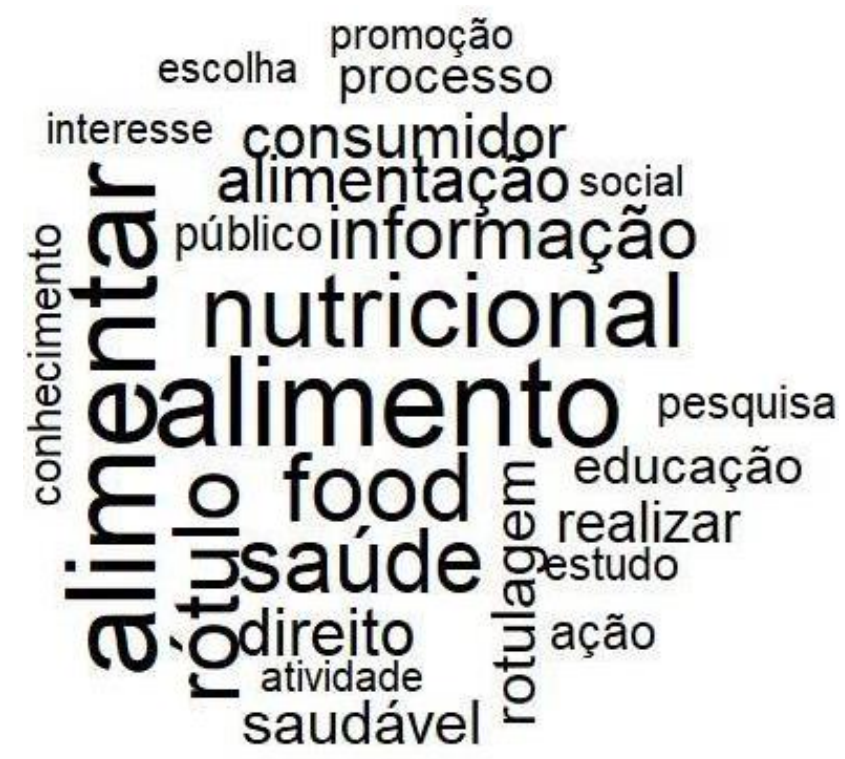

Figura 3 - Nuvem de palavras

Goiânia, 2020. Fonte: Elaborada pelos autores 
Nessa análise, utilizou-se também como padrão somente as palavras com frequência maior que sete. Na Figura 3, é possível perceber que as palavras que mais se destacaram foram: alimentar, alimento, nutricional, food, saúde, rótulo, informação, alimentação e consumidor.

\section{Considerações Finais}

O objetivo deste artigo foi compreender, a partir de um recorte, como o assunto rotulagem foi abordado por pesquisas a partir de 2015. Com base nos artigos encontrados e selecionados, percebeuse que há crescente interesse pelo assunto, com o aumento do número de publicações, especialmente em 2017.

A maior dificuldade encontrada para selecionar os artigos se dá, pelo fato de que boa parte do número de pesquisas que abrangem a área de rotulagem, não se concentrarem somente nesse assunto. O tema está sempre ligado a alguma outra área como agrotóxicos, alimentos transgênicos e orgânicos e a parte de rotulagem ocupa espaço muito ínfimo na pesquisa, o que não confere profundidade ao objeto de estudo.

Essa percepção também pode explicar o baixo número de publicações selecionadas para análise, visto que, o objetivo era compreender o cenário da pesquisa das políticas públicas de rotulagem na segurança alimentar e nutricional, não sendo pertinente abordar outras áreas. Esse fato pode ser considerado uma limitação deste estudo.

No momento em que esse estudo foi feito, a ANVISA contava com uma consulta pública em aberto sobre novas medidas para a rotulagem brasileira, o que reforçou os resultados levantados por esse estudo. Cabe destacar que se a rotulagem dos alimentos, ao orientar o consumidor sobre a qualidade e a quantidade dos constituintes nutricionais dos produtos, pode promover escolhas alimentares apropriadas, é muito importante que os consumidores tenham o hábito de analisar rótulos (Câmara e Cols, 2008). Esse aspecto não parece ser algo cultural no âmbito de consumo de alimentos no Brasil. Mesmo considerando ser fidedignas as informações nutricionais, não há um costume de interpretar a combinação de nutrientes em cada rótulo. Cerca de $43 \%$ dos consumidores brasileiros, no ato da compra dos alimentos, buscam nas embalagens informações sobre os benefícios para a saúde (Coutinho, 2004).

Mesmo que haja uma busca efetiva pela informação contidas nos rótulos, uma parcela inferior a $20 \%$ dos alimentos pode ser considerada conforme (Smith \& Muradian, 2011). Nessa direção, os dados aqui apresentados corroboram que há margem para o crescimento do estudo da área de rotulagem, voltada para a segurança alimentar e nutricional, com foco na promoção de novas políticas públicas, principalmente àquelas que possam combater as doenças crônicas não transmissíveis como a obesidade e a desnutrição. 


\section{Referências Bibliográficas}

Abramovay, Ricardo. (2017). O que é fome. São Paulo: Brasiliense.

Alcantara, Felipe B., Rodrigues, Jessica C. S., Silva, Khetlin K., Santos, Mariana C., Silva, Thainá M., \& Berni, Andrea L. (2018). A Influência da mídia e publicidade na alimentação de escolares: o papel da educação alimentar. Revista Eletrônica Acervo Saúde. e. 11(13), 1-10. Acessado em 30 de setembro de 2020, de: https://doi.org/10.25248/reas.e1005.2019

Aliaga, Marie Agnes e Cols. Avaliação participativa da segurança alimentar e nutricional em uma comunidade de Salvador, Brasil. Ciência \& Saúde Coletiva, 25, 2595-2604, 2020. http://dx.doi.org/10.1590/1413-81232020257.25252018

Almeida , Jessica Yoanna Lima de. e Cols. (2018). Addressing healthy food with children in a public school: a report of experience, ReonFacema, 4(4), 1351-1355. Acessado em 12 de janeiro de 2020, de: https://www.facema.edu.br/ojs/index.php/ReOnFacema/article/download/345/277

Assao, Tatiana Y., Cordeiro, Amábela A., Costa, Christiane \& Cervato, Ana Maria. (2007). Práticas e percepções acerca da segurança alimentar e nutricional entre os representantes das instituições integrantes de um centro de referência localizado na região do Butantã, município de São Paulo. Saúde e Sociedade, 16(1), 102-116. Acessado em 30 de setembro de 2020, de: http://dx.doi.org/10.1590/S0104-12902007000100010

Belik, Walker. (2003). Perspectivas para segurança alimentar e nutricional no Brasil Prospects for food and nutricional safety in Brazil. Saúde e Sociedade, 12(1), 12-20. Acessado em 30 de setembro de 2020, de: http://www.scielo.br/pdf/sausoc/v12n1/04.pdf

Bevilacqua, Solon, \& Villena, John E. N. (2019). Política Pública, Autocontrol y Tendencia Intertemporal en el Control de la Obesidad y Sobrepeso. Revista Gestão \& Políticas Públicas, 9(2), 184-197. Acessado em 24 de Abril de 2020, de: https://doi.org/10.11606/rgpp.v9i2.168659

Brasil. (1969, 21 de outubro). Decreto-Lei no 986. Institui normas básicas sobre alimentos. Brasília: Imprensa Nacional.

Brasil. (1988). Constituição da República Federativa do Brasil de 1988. Brasília, DF: Presidência da República. Acessado em 04 de novembro de 2020, de: http://www.planalto.gov.br/ccivil 03/constituicao/constituicao.htm

Brasil. (1990, 11 de setembro). Lei $n^{\circ}$ 8.078. Dispõe sobre a proteção do consumidor e dá outras providências. Diário Oficial da União, Brasília, DF. Acessado em 04 de novembro de 2020, de: http://www.planalto.gov.br/ccivil 03/leis/18078compilado.htm

Brasil. (2002, 20 de setembro). Resolução - RDC $n^{\circ}$ 259. ANVISA. Aprovar o Regulamento Técnico sobre Rotulagem de Alimentos Embalados. Brasília, DF. Acessado em 04 de novembro de 2020, de: $\quad$ https://www.gov.br/agricultura/pt-br/assuntos/inspecao/produtos-vegetal/legislacao1/biblioteca-de-normas-vinhos-e-bebidas/resolucao-rdc-no-259-de-20-de-setembro-de2002.pdf/view

Brasil. (2003, 23 de dezembro). Resolução - RDC $n^{\circ}$ 360. ANVISA. Aprovar o Regulamento Técnico sobre Rotulagem Nutricional de Alimentos Embalados. Brasília, DF. Acessado em 04 de novembro de 2020, de: http://bvsms.saude.gov.br/bvs/saudelegis/anvisa/2003/rdc0360_23_12_2003.html

Brasil. (2006a, 15 de setembro). Lei $n^{\circ}$ 11.346. Cria o Sistema Nacional de Segurança Alimentar e Nutricional - SISAN. Diário Oficial da União, Brasília, DF. Acessado em 04 de novembro de 2020, de: http://www.planalto.gov.br/ccivil_03/_ato2004-2006/2006/lei/111346.htm 
Brasil. (2006b). Ministério da Saúde. Secretaria de Vigilância em Saúde. Secretaria de Atenção à Saúde. Política Nacional de Promoção da Saúde. Brasília: Ministério da Saúde. (Série B, Textos Básicos de Saúde).

Brasil. (2007, 06 de dezembro). Decreto Presidencial $n^{\circ}$ 6.286, de 5 de dezembro de 2007. Institui o Programa Saúde na Escola (PSE), e dá outras providências. Diário Oficial da União.

Brasil. (2014). Ministério da Saúde. Secretaria de Atenção à Saúde. Departamento de Atenção Básica. Guia alimentar para a população brasileira. Brasília: Ministério da Saúde. Acessado em 24 de Março de 2020, de: https://bvsms.saude.gov.br/bvs/publicacoes/guia alimentar_populacao brasileira 2ed.pdf

Boog, Maria C. F. (1999). Educação nutricional em serviços públicos de saúde. Cadernos de Saúde Pública, 15. Acessado em 24 de Março de 2020, de: https://doi.org/10.1590/S0102$\underline{311 X 1999000600014}$

Brugué, Quim., \& Tarragó, Daniel. (2014). La administración deliberativa: de la eficacia y la eficiencia a la inteligencia, y de la burocracia a la innovación. Em Cardoso Júnior, J. C. (Org.). PPA 2016-2019: inovação institucional e revolução administrativa. Brasília: Ipea.

CAISAN. (2018). Câmara Interministerial de Segurança Alimentar e Nutricional. Plano Nacional de Segurança Alimentar e Nutricional: 2016/2019. Brasília, DF: CAISAN. Acessado em 10 de novembro de 2020 ,

de: http://www.mds.gov.br/webarquivos/arquivo/seguranca alimentar/caisan/Publicacao/Caisan Nacio nal/PLANSAN\%202016-2019_revisado_completo.pdf

Câmara, Maria C. C., Marinho, Carmem L. C., Guilam, Maria C., \& Braga, Ana M. C. B. (2008). A produção acadêmica sobre a rotulagem de alimentos no Brasil. Revista Panam Salud Publica, 23(1), 52-58. Acessado em 04 de novembro de 2020, de: https://www.scielosp.org/pdf/rpsp/2008.v23n1/52-58

Carvalho, Gabriel R., Gandra, Fernanda P. P., Pereira, Rafaela C., Dias, Ludmila B., \& Pereira, Michel C. A. (2019). Percepção sobre mídia e comportamento na compra de alimentos: estudo com consumidores de dois municípios do sul de minas gerais. Brazilian Journal of Food Technology, 22, 1-8. Acessado em 04 de novembro de 2020, de: https://doi.org/10.1590/1981-6723.17018

Castro, Inês Rugani Ribeiro de. (2019). A extinção do Conselho Nacional de Segurança Alimentar e Nutricional e a agenda de alimentação e nutrição. Cad. Saúde Pública, 35 (2), 1-4. Acessado em 24 de Março de 2020, de: https://doi.org/10.1590/0102-311X00009919

Coutinho, Janine G., \& Recine, Elisabete. (2007). Experiências internacionais de regulamentação das alegações de saúde em rótulos de alimentos. Revista Panamericana de Salud Publica, 22, 432-437. Acessado em 24 de Março de 2020, de: https://scielosp.org/article/rpsp/2007.v22n6/432-437/pt/

Dantas, Rafaela R., \& Silva, Giselia A. P. D. (2019). O papel do ambiente obesogênico e dos estilos de vida parentais no comportamento alimentar infantil. Revista Paulista de Pediatria, 37(3), 363-371. Acessado em 06 de novembro de 2020, de: https://doi.org/10.1590/1984-0462/;2019;37;3;00005

Medeiros, Ivan L., Vieira, Alessandro., Braviano, Gilson., \& Gonçalves, Berenice. (2015). Revisão Sistemática e Bibliometria facilitadas por um Canvas para visualização de informação. InfoDesignRevista Brasileira de Design da Informação, 12(1), 93-110. Acessado em 30 de setembro de 2020, de: https://www.infodesign.org.br/infodesign/article/view/341

Dutra, Gabriela., \& Malagoli, Leticia (2019). A construção de um hábito alimentar saudável desde a educação infantil. Revista GepesVida, 5(10). 
Erdmann, Alacoque L., Marziale, Maria Helena., Pedreira, Mavilde da luz., Lana, Francisco., Pagliuca, Lorita., Padilha, Maria I., \& Fernandes, Josicelia D. (2009). A avaliação de periódicos científicos qualis e a produção brasileira de artigos da área de enfermagem. Revista LatinoAmericana de Enfermagem, 17(3). Acessado em 10 de março de 2020, de: http://www.redalyc.org/articulo.oa?id=281421908019

Fernandes, Nadia R., \& Silva, Izabel C. R. (2012) Rotulagem Nutricional: um importante instrumento de educação do consumidor? Vigilância Sanitária, p. 12.

Gomes, Mônica A., \& Pereira, Maria L. D. (2005). Família em situação de vulnerabilidade social: uma questão de políticas públicas. Ciência \& Saúde Coletiva, 10, 357-363.

Gonçalves, Maetê P., Campos, Silvana T. D., \& Sarti, Flávia M. (2011). Políticas públicas de segurança alimentar no Brasil: uma análise do Programa de Restaurantes Populares. Revista Gestão \& Políticas Públicas, 1(1), 92-111. Acessado em 10 de março de 2020, de: https://www.revistas.usp.br/rgpp/article/view/97826

Jaime, Patrícia Constante. (2020). Pandemia de COVID19: implicações para (in) segurança alimentar e nutricional.

Landim, Liejy., Cordeiro, M.araysa., Barbosa, Amanda., Severo, Juliana., Ibiapina, Daniela., \& Pereira, Brenda. (2020). Avaliação nutricional, consumo alimentar e frequência de ultraprocessados em escolares da rede pública. Revista Eletrônica Acervo Saúde, 12(5), e2427-e2427. Acessado em 10 de março de 2020, de: https://doi.org/10.25248/reas.e2427.2020

Lopes, Wanessa C., Pinho, Lucinéia D., Caldeira, Aantônio P., \& Lessa, Angelina D. C. (2020). Consumo de alimentos ultraprocessados por crianças menores de 24 meses de idade e fatores associados. Revista Paulista de Pediatria, 38. Acessado em 10 de março de 2020, de: https://doi.org/10.1590/1984-0462/2020/38/2018277

Machado, Mick., Gabriel, Cristine., Soar, Claudia., Mamed, Gisele., Machado, Patricia M. D. O., Lacerda, Josimari., Martins, Milena., \& Marcon, Maria. (2018). Adequação normativa dos planos estaduais de segurança alimentar e nutricional no Brasil. Cadernos de Saúde Pública, 34, e00206716. Acessado em 10 de março de 2020, de: https://doi.org/10.1590/0102-311X00206716

Monteiro, Renata., Coutinho, Janine., \& Recine, Elisabetta. (2005). Consulta aos rótulos de alimentos e bebidas por freqüentadores de supermercados em Brasília, Brasil. Revista Panamericana de Salud Pública, 18, 172-177. Acessado em 10 de março de 2020, de: https://www.scielosp.org/pdf/rpsp/2005.v18n3/172-177

Melo, Marcus A. (1999). Estado, governo e políticas públicas. O que ler na ciência social brasileira (1970-1995), 3, 59-100. In: MICELI, S. (org.). O que ler na Ciência Social Brasileira (1970-1995): Ciência Política. São Paulo/ Brasília: Sumaré/Capes.

Minuzi, Gabrielle A., \& Pommer, Roselene M. G. (2019). Reflexões iniciais sobre a alimentação das classes sociais. RELACult-Revista Latino-Americana de Estudos em Cultura e Sociedade, 5(4). Acessado em 30 de março de 2020, de: https://doi.org/10.23899/relacult.v5i4.1198

Patzlaff, M. E. B., \& Melo, S. S. M. (2020). Informações nutricionais nos rótulos de alimentos industrializados em relação às necessidades nutricionais de pré-escolares. Brazilian Journal of Health Review, 3(6), 17952-17965.

Pereira, Mônica C. S., Jesus, Maria C. P., Vassimon, Helena S., Nascimento, Lilian C. G., \& Tavares, Maria F. L. (2019). Direito do consumidor às informações nos rótulos dos alimentos: perspectiva de profissionais envolvidos em políticas públicas. Aletheia, v. 52, n. 1, p. 85- 101. 
Silva, Amanda M. P. D., \& Senger, Maria H. (2014). A informação nutricional na rotulagem obrigatória dos alimentos no Brasil: percepções sobre fatores motivadores e dificultadores de sua leitura e compreensão. Resultados de um estudo exploratório com grupos focais. Nutrire Rev. Soc. Bras. Aliment. Nutr, 327-337.

Smith, Ana C. D. L., \& Muradian, Ligia B. D. A. (2011). Rotulagem de alimentos: avaliação da conformidade frente à legislação e propostas para a sua melhoria. Revista do Instituto Adolfo Lutz (Impresso), 70(4), 463-472. Acessado em 24 de Março de 2020, de:

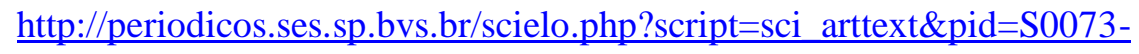
$\underline{98552011000400004 \& \operatorname{lng}=\mathrm{pt} \& \mathrm{nrm}=\mathrm{iso}}$

Souza, Celina. (2003). "Políticas Públicas: Questões Temáticas e de Pesquisa", Caderno CRH 39: 1124.

Souza, Luana C. A., Santos, V. D. L., Oliveira, R. A. D., Félis, K. C., Santos, C. C. T., \& Moraes Filho, I. M. D. (2019). Perfil nutricional de pré-escolares do programa mais educação na cidade de Goiânia-GO. Revista de Divulgação Científica Sena Aires, 8(1), 36-48.

Vasconcellos, Ana Beatriz., \& Moura, Leide. (2018). Segurança alimentar e nutricional: uma análise da situação da descentralização de sua política pública nacional. Cadernos de Saúde Pública, 34, e00206816. Acessado em 24 de Março de 2020, de: https://doi.org/10.1590/0102-311X00206816

Versiani, Rodrigo. (2020). O controle da publicidade infantil de alimentos: hermenêutica à luz do direito fundamental de proteção à criança com absoluta prioridade. Dissertação (Mestrado). Universidade Federal de Uberlândia, Brasil. Acessado em 24 de Março de 2020, de: http://doi.org/10.14393/ufu.di.2020.712

Zanini, Roberta., Muniz, Ludimila., Schneider, Bruna., Tassitano, Rafael., Feitosa, Wallacy., \& González-Chica, David. (2013). Consumo diário de refrigerantes, doces e frituras em adolescentes do Nordeste brasileiro. Ciencia \& saude coletiva, 18(12), 3739-3750. Acessado em 24 de Março de 2020, de: https://doi.org/10.1590/S1413-81232013001200030

Recebido em 18/02/2020. 